KOMPASS

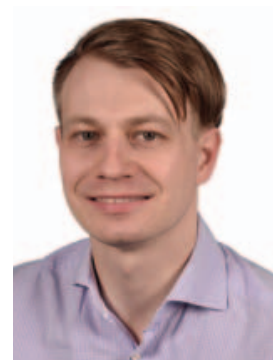

Sebastian Zimmer

Koordination «DermaCampus»

\title{
Sorgen, Rechte und Pflichten im Praxisalltag
}

Steigende Patientenzahlen und immer mehr administrative Anforderungen verdichten die ärztliche Arbeit und setzen uns Ärzte unter immer größeren Druck. Der hilflose Helfer bleibt jedoch ein Tabuthema. Im Interview mit Dr. Bernhard Mäulen, Psychiater und Betreiber der Internetseite www.aerztegesundheit.de, werden Gründe für Depression und Suizidalität unter Ärzten beleuchtet und unterstützende Hinweise gegeben.

Eine Schwangerschaft dürfte für viele das Gegenteil von Trauer bedeuten und im Leben äußerst sinnstiftend sein, jedoch wirft sie auch organisatorische Fragen auf, insbesondere im Kontext der Regelungen ärztlicher Weiterbildung. Einen Einblick in die Rechte einer werdenden Mutter und Ärztin gibt uns der Beitrag zur «Schwangerschaft in der Weiterbildung».

Jeder Arzt kennt die Situation, in der Angehörige über den Zustand des Patienten Informationen erhalten wollen, doch hierzu bedarf es einer expliziten Zustimmung des Patienten. Über Situationen, in denen die Schweigepflicht umgangen werden darf oder gar muss, wird im Beitrag von Henning Kropp und Uwe Günther detailliert berichtet.

Die Komposition und Koordination von Informationen ist auch Thema des letzten Beitrags: Wir leben in einer Zeit, in der wir vermeintlich jederzeit und quasi überall auf jede gesuchte Information zugreifen können. Brauchen wir dann noch Verlage? Hierfür vermitteln die Geschäftsführerin des Karger Verlags Gabriella Karger, wir als Nutzer von Verlagsdiensten sowie wichtige Vertreter der Medienlandschaft Eindrücke aus unterschiedlichen Perspektiven.

Wir wünschen Ihnen eine anregende Lektüre! Mit besten Grüßen,

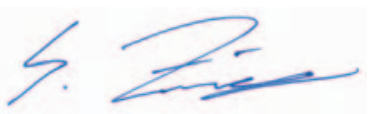

\section{KARGER}

() 2018 S. Karger GmbH, Freiburg

Fax +497614520714

information@karger.com www.karger.com
Dr. Sebastian Zimmer

Universitätsmedizin der Johannes Gutenberg

Universität Mainz

Hautklinik und Poliklinik, Geb. 401, CRC

Langenbeckstr. 1, 55131 Mainz, Deutschland 


\section{Ärztegesundheit}

Sucht, Selbstmedikation und Suizidalität bei Ärzten

«Ich werde auf meine eigene Gesundheit, mein Wohlbefinden und meine Fähigkeiten achten, um auf höchstem Niveau zu behandeln». Dies ist eines von 13 Leitmotiven der Genfer Deklaration des Weltärztebundes und Teil der Berufsordnung deutscher Ärzte. Häufig fällt es den Ärzten jedoch schwer, ihre eigene Gesundheit bei der Pflege ihrer Patienten zu berücksichtigen. Die hohe Erwartungshaltung der Patienten aber auch sich selbst gegenüber sowie zeitliche und psychische Überlastung schlagen sich immer häufiger in seelischen Erkrankungen und Sucht nieder, die schwer wiegen und gar zum Freitod führen: Medizinerlnnen weisen empirisch belegt eine bis zu 3,4-fach höhere Suizidrate auf als die Allgemeinbevölkerung [1].

Dr. Bernhard Mäulen, Facharzt für Psychiatrie \& Psychotherapie, interessiert sich bereits seit Jahrzehnten für die seelischen Belange seiner ärztlichen Kollegen. Im Campus erörtert er die Gründe psychischer Überlastung bei Medizinern und schildert seine Erfahrungen mit Ärzten zwischen Erschöpfung, Doping und Verzweiflung. Mit umsichtigen Empfehlungen schafft er schließlich Aussichten auf Licht am Ende des Tunnels.

Depressionen, Suizid und Suizidalität bei ÄrztInnen: Gab es das schon immer?

In Ärztebiografien finden Sie immer wieder überraschende Hinweise darauf - sogar schon um das Jahr 1900. Statistisch sind Depressionen, Suizid und Suizidalität unter MedizinerInnen jedoch erst in den 1970er bzw. 1980er Jahren erfasst worden. Aber das Thema war immer da und immer schon schambesetzt. Aufgrund der Bereitschaft von US-Kollegen, sich hier zu öffnen, konnten auch bei uns betroffene KollegInnen anfangen, darüber zu schreiben und zu reden, was nach und nach die Größe des Problems verdeutlichte. Bei Betroffenen handelt es sich nicht um «schwarze Schafe» oder schwache Mitglieder des Berufsstands, sondern eine derartige Erkrankung trifft viele im Laufe ihrer ärztlichen Berufskarriere.

Würden Sie sagen, dass Suizidalität unter ÄrztInnen noch mehr stigmatisiert wird als in der übrigen Bevölkerung?

Ja, das würde ich. Lange hatten wir den überhöhten Anspruch: «Wenn die anderen nicht mehr können, machen wir weiter.» Gerade die «hilflosen Helfer» [2] haben Schwächen und ihre eigene Betroffenheit. Es ist ein wichtiger Teil der beruflichen Wahrheit, das anzuerkennen und offen damit umzugehen.

\section{Unterscheiden sich die Gründe für Selbstmord bei ÄrztInnen von denen in anderen Berufsgruppen?}

Die Gründe sind genau die gleichen: Depression, Suchterkrankungen, partnerschaftliche Krisen und Ähnliches; auch Psychosen, d.h. Krankheiten, die statistisch nachweisbar mit einer erhöhten Suizidalität einhergehen, treffen ÄrztInnen und zeigen die gleiche Symptomatik. Trotzdem gibt es eine nachweisbare, deutlich erhöhte Suizidrate bei Ärztlnnen [3].

Es gibt zwar keinen Beweis dafür, aber viele Autoren stimmen über- ein, dass die Fähigkeit der Helfer, selbst Hilfe anzunehmen, im Vergleich mit der Allgemeinbevölkerung weit unterdurchschnittlich ist. Dabei gilt für Depressionen wie für Sucht: Wer sich nicht behandeln lässt, wird auch nicht gesund! Außerdem sind wir nicht gerade offen auf die Betroffenen aus den eigenen Reihen zugegangen. Es brauchte Jahrzehnte, bis eine neue Kultur gewachsen ist, die das zuließ.

Immer wieder erkranken die besonders Engagierten und Aufopferungsvollen, wie wir das auch vom Burnout kennen. Also, die, die angetrieben sind durch ein hohes Ich-Ideal, die sich eher wenig verzeihen, die eher selten sagen: «lch bin auch mal dran und jetzt ist hier genug».

\section{In der Ärzteschaft scheint es auffällig zu sein, dass sich besonders} viele Ärztinnen das Leben nehmen. Ist das so?

Ja, aber auch dazu gibt es keine belastbaren Daten. Für Frauen galten keine höheren Maßstäbe als für Männer. Aber Frauen waren in der Medizin lange eine Minderheit, die sich in einer Männerdomäne behaupten musste. Das hat sicherlich dazu geführt, dass mehr Biss und Härte erforderlich waren, um überhaupt Fuß zu fassen. Entsprechend schwieriger könnte es für diese Frauen gewesen sein, bei eigener Krankheit Hilfe einzufordern. Zudem kann es aufgrund der Partnerwahl dieser Frauen dazu kommen, dass sie auch zu Hause in einer starken oder überlegenen Rolle gefangen sind und aus dieser nicht so einfach aussteigen können. Aber das sind Vermutungen aus meinem Praxisalltag.

Betroffene Medizinerlnnen nehmen deutlich weniger Antidepressiva als Betroffene in anderen Berufsgruppen, dafür deutlich mehr Psychopharmaka wie Tranquilizer, Antipsychotika - woher kommt das?

Das ist leider so. Es gibt einige Untersuchungen [4] zur Selbstmedikation bei Ärztlnnen. Bei einer Kopfschmerztablette ist das vielleicht nicht bedenklich, aber wenn es um Antidepressiva oder Antipsychotika geht, ist Selbstmedikation ein absolutes No-Go - trotzdem kommt es vor. In Peer-Überprüfungen der Selbstmedikation von Ärzten wurde die Fehlerhaftigkeit bei der Selbstmedikation belegt [5]. Es besteht das Risiko, eine falsche Substanzklasse oder Dosis einzunehmen.

\section{Welche Rolle spielen dabei Drogen im Allgemeinen, z.B. Alkohol?}

«Wer Sorgen hat, hat auch Likör», hieß es früher. Alkohol als gesellschaftlich verfügbare und legitime Droge ist auch für uns Ärzte das Suchtmittel erster Wahl, um Stress, Sorgen und Überlastung zu begegnen und vielleicht ein Stück Ausgleich zu schaffen. Für diejenigen, die prädisponiert für eine Suchterkrankung sind, kann dies der Anfang für eine Suchtgeschichte sein, und alle Suchterkrankungen haben eine erhöhte Suizidgefahr. Bei ÄrztInnen kommt hinzu, dass sie sich zusätzlich noch Medikamente verschreiben können, die sonst nicht verfügbar sind. 
Ist die Situation eher ein gesellschaftliches Problem oder auch ein Problem des Gesundheitssystems?

Es ist kein Entweder-oder, sondern ein Sowohl-als-auch. Jede/n, die/der in einer stressreichen Umgebung arbeitet, was auf den Medizinberuf zutrifft, ist der Gefahr einer Suchtkrankheit oder eines Burnouts ausgesetzt. Zudem beschäftigt sich die heutige Ärztegeneration viel ausgiebiger mit administrativen Aufgaben. Die Zeit dafür wird häufig als verloren erlebt und ruft nachhaltige Frustration und Ohnmachtserleben hervor. Zusammen mit Zeitnot ist das ein erheblicher chronischer Belastungsfaktor.

Die meisten Ärztlnnen mögen ihren Beruf, und sie werden gerne anständig dafür bezahlt - es gab Zeiten, da verdienten Mediziner weit weniger als jetzt. Die direkte Begegnung mit den Patienten ist unsere Berufung, aber die fachfremden Tätigkeiten, die bürokratischen, abrechnungsbedingten oder technischen Hintergrund haben, werden nicht als produktiv wahrgenommen, nehmen jedoch sehr viel Zeit in Anspruch. Wahrscheinlich hat die vorherige Ärztegeneration genauso viele Patientlnnen behandelt und rein zeitlich genauso viel gearbeitet wie die heutige. Aber sie erlebten sich in ihrem Beruf als deutlich freier. Zwar gab es auch da ausgebrannte, suchtkranke, suizidale Ärzte, aber die aktuelle Burnout-Rate ist sehr hoch.

Erschwerend kommt hinzu, dass heutzutage die Krankenhäuser unterfinanziert sind und es keine geregelte Vertretung gibt: Es müssen immer alle da sein, damit der Laden läuft. Man mutet den anderen im Team mit einer krankheitsbedingten Abwesenheit sehr viel zu. Allerdings ist es paradox: Wenn ich mir beim Skifahren eine Verletzung zuziehe und erst nach zwei Monaten humpelnd wiederkomme, ist das völlig akzeptiert. Wenn ich aber we-

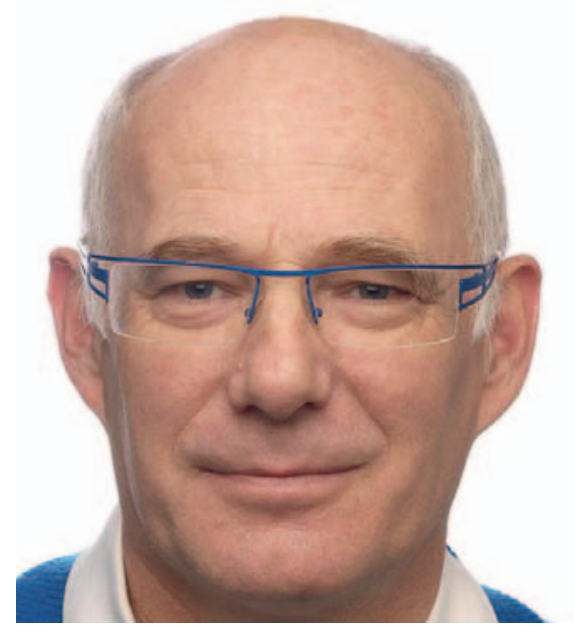

«Selbstbehandlung ist ein Kunstfehler!»
Auch auf Fachkonferenzen und in der Fachliteratur wird das Thema Ärztegesundheit diskutiert. Deswegen bin ich zuversichtlich, dass die jüngeren Kolleglnnen theoretisch um die Gefahr wissen.

Der schwierige Faktor ist nach wie vor, ob und wann der Betroffene bereit ist, sich Hilfe zu holen. Das ist noch immer das Nadelöhr! Es gibt hier langsam einen Einstellungswandel. Aber bis betroffene ÄrztInnen tatsächlich zeitnah Unterstützungsangebote in Anspruch nehmen, gibt es noch einiges zu tun.

\section{Was würden Sie jungen/angehenden Ärztlnnen raten, um ange-}

messen mit psychischen Belastungen umzugehen?

Den jüngeren ÄrztInnen möchte ich sagen: Selbstbehandlung ist ein Kunstfehler! Die Medizin ist ein hochstressiger Beruf. Als angehende/r Medizinerln müssen Sie damit rechnen, dass Sie in Ihrem Beruf mindestens einmal in Ihrem Leben verklagt werden, ausbrennen oder in eine persönliche Krise geraten. Das wird die Regel sein und nicht die Ausnahme! Und es ist wichtig, dass Sie für diese Fälle vorsorgen.

Aufgrund meiner persönlichen Erfahrung würde ich das noch etwas härter formulieren: Dem System ist es ziemlich egal, wie es der/dem einzelnen Ärztin/Arzt geht. Dir darf es aber nicht egal sein! Du bist dein erster Patient! Wieso hast du denn diesen Beruf erlernt? Gilt Mitgefühl und Fürsorge nur für die anderen? Medizinern wird der Grundsatz nihil nocere («auf keinen Fall schaden») $\stackrel{\Xi}{\bar{\sigma}}$ mit auf den Weg gegeben. Wenn wir den auf uns anwenden, kann man sich fragen, ob man einen Job annehmen sollte, der uns voraussichtlich und mit hoher Wahrscheinlichkeit hoch belastet und manchmal unsere Gesundheit zerstört. Aber wenn ich mich schon fürs Arztsein entscheide, muss ich von Anfang an protektive Faktoren, einen gen eines Suizidversuchs, einer Sucht oder einer Depression entsprechend lange weg bin, wird danach entweder nicht darüber gesprochen oder die Arbeitsfähigkeit in Frage gestellt. Da haben wir leider immer noch, auch unter ÄrztInnen, zwei Maßstäbe für somatische und psychiatrische Erkrankungen.

Heute fühlen sich ein Drittel der ÄrztInnen gegängelt und in einem System gefangen, das die freie ärztliche Entscheidung einschränkt. Ich glaube schon, dass dies von den äußeren situativen Faktoren mit der entscheidende ist. Aber es müssen auch individuelle Faktoren berücksichtigt werden.

Was könnte man dem Problem der Suizidalität bei ÄrztInnen konkret entgegensetzen?

Das ist mittlerweile gut erforscht und wird bereits in der Ausbildung thematisiert: ein offener Umgang mit dem Thema, die Berücksichtigung der Stressbelastung, die eigene gesundheitliche Gefährdung, aber auch die Notwendigkeit, sich frühzeitig Hilfe zu holen. Wie bei den meisten großen Veränderungen braucht es aber fast eine Generation, bis ein Umdenken einsetzt und sich stabilisiert.
Selbstschutz, einbauen, z.B. eine gute Ausbildung, genügend Urlaub, in Zwischenphasen gegebenenfalls ein Sabbatical, ein positives Netzwerk von KollegInnen und Unterstützern, Supervisionen, Entspannungsverfahren, und wenn nötig, hilft Coaching oder eine stützende, ambulante Psychotherapie.

\section{Dr. Mäulen, wir danken Ihnen sehr für das offene Gespräch!}

Fast sein ganzes Berufsleben als Arzt hat sich Dr. Bernhard Mäulen nicht nur für das Wohlbefinden der Patientlnnen, sondern auch für deren Behandler, nämlich ÄrztInnen und TherapeutInnen, interessiert. Nachdem er sich als Psychiater niedergelassen hatte, gründete er vor über 20 Jahren die Internetseite www.aerztegesundheit.de, um die zahlreichen Anfragen von KollegInnen für Beratungstermine, aber auch Presseanfragen zu bewältigen und die Gesundheit von Ärzten stärker ins Bewusstsein zu rücken. Die Plattform wird heute von bis zu 100000 Userlnnen jährlich besucht. 
1 Reimer C, Trinkaus S, Jurkat HB: Suizidalität bei Ärztinnen und Ärzten. Psychiat Prax 2005;32:381-385

2 Schmidbauer W: Die hilflosen Helfer: Über die seelische Problematik der helfenden Berufe. Reinbeck, Rowohlt, 1978

3 Mäulen, B: Jedes Leben zählt - Suizid von Anästhesisten. Der Anaesthesist 2010:59;395-400.

4 Montgomery AJ, Bradley C, Rochfort A, Panagopoulou E: A review of self-medication in physicians and medical students. Occup Med (Lond) 2011;61:490-497.
5 Chambers R, Belcher J: Self-reported health care over the past 10 years: a survey of general practitioners. Br J Gen Pract 1992;42:153-156.

Kontaktadresse: Dr. Bernhard Mäulen, Arzt für Psychiatrie und Psychotherapie, Institut für Ärztegesundheit, Vöhrenbacherstraße 4, 78050 Villingen, Deutschland,docmaeulen@gmail.com.

\section{Arbeitsrecht}

\section{Schwangerschaft in der Weiterbildung}

Viele junge Ärztinnen machen ihre Facharztweiterbildung im Alte zwischen 25 und 35 Jahren, also genau dann, wenn oftmals die Familienplanung konkret wird. Kliniken geraten immer mehr unter Druck, familienfreundliche Lösungen wie Teilzeit, Gleitzeit oder Kinderbetreuung anzubieten, weil derzeit 60\% der Medizinstudierenden Frauen sind und es folglich immer mehr Ärztinnen geben wird. Bei vielen Kliniken lassen familienfreundliche Maßnahmen aber noch zu wünschen übrig. Deshalb sollte man bei der Bewerbung berücksichtigen, inwiefern der potenzielle Arbeitgeber mit einer Halbtagstätigkeit, mit einem Betriebskindergarten etc. entgegenkommt. Was ist bei einer Schwangerschaft und der Elternzeit zu beachten?

\section{No-Gos in der Schwangerschaft}

Es gibt verschiedene gesetzliche Regelungen, die schwangere Ärztinnen und ihr ungeborenes Kind vor gesundheitlichen Schäden und Überforderung am Arbeitsplatz schützen sollen. Insbesondere im Mutterschutzgesetz und in der Mutterschutzrichtlinienverordnung ist festgelegt, was man als werdende oder stillende Mutter nicht mehr machen darf. Dazu gehört u.a.:

- Nachtarbeit zwischen 20:00 und 6:00 Uhr

- Bereitschaftsdienste

- Notdienste

- Mehrarbeit

- regelmäßiges Heben von mehr als $5 \mathrm{~kg}$

- Kontaktzu giftigen und gesundheitsschädlichen Gefahrenstoffen

- Kontakt zu potenziell infektiösem Material

- Injektionen, Punktionen, Blutentnahmen, Operationen.

Es empfiehlt sich, die Schutzverordnungen genau durchzulesen, damit man weiß, auf welche Rechte man sich berufen kann. So ist der Arbeitgeber z.B. auch verpflichtet, einer schwangeren Ärztin einen Ruheraum zur Verfügung zu stellen und die Schwangerschaft beim zuständigen Landesamt für Arbeitsschutz und Ge-

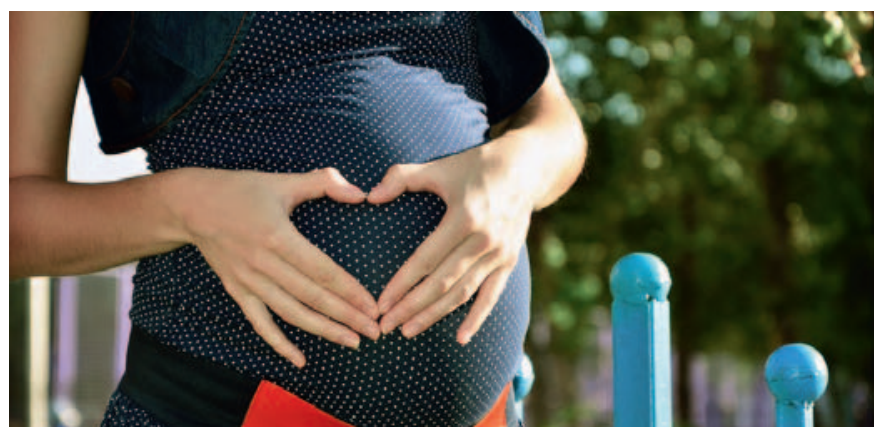

sundheitsschutz zu melden. Kann der Arbeitgeber nicht alle Maßnahmen umsetzen, die einer schwangeren Ärztin zustehen, muss der Betriebsarzt ein Beschäftigungsverbot bescheinigen. Man bleibt dann zu Hause, bezieht das Gehalt aber weiter.

Die Richtlinien zum Schutze von Mutter und Kind werden aber auch kritisiert, z.B. vom Deutschen Ärztinnenbund (DÄB), weil der schwangeren Ärztin der Patientenkontakt aufgrund der Bestimmungen mehr oder weniger verboten wird. Die Bestimmungen sind z.T. schon 20 Jahre alt, die OP-Technik hat sich in dieser Zeit aber stark weiterentwickelt. Das in der Richtlinie festgelegte generelle OP-Verbot erscheint vielen als zu hart. Derzeit haben schwangere Ärztinnen nur zwei Möglichkeiten: Entweder sie teilen ihre Schwangerschaft frühzeitig mit. Dann müssen sie damit rechnen vom Dienst freigestellt zu werden und können keine weiteren Leistungen für die Weiterbildung sammeln. Oder: Sie machen die Schwangerschaft erst sehr spät öffentlich. Dann genießen sie keinen gesonderten Schutz und werden von den unwissenden Kollegen womöglich wegen häufiger Ruhezeiten kritisiert.

\section{Mutterschutzbestimmungen}

Bis es zu einer überarbeitenden Mutterschutz-Richtlinie für Ärztinnen kommt, muss man als schwangere Ärztin also selbst überlegen, wie man mit der eigenen Schwangerschaft umgeht. Am besten bespricht man sich gründlich mit dem Partner und der Frauenärztin (auch sie darf ein Berufsverbot aussprechen). Oft sagt einem auch der eigene Körper, was er braucht.

Wenn man in der Schwangerschaft arbeitet, besteht die Möglichkeit, sechs Wochen vor dem errechneten Geburtstermin in den «Mutterschutz» zu gehen, also nicht mehr zu arbeiten. Nach der Geburt ist man 8 Wochen verpflichtend im Mutterschutz. Während dieser Zeit bekommt man ein Mutterschaftstagegeld von der Krankenkasse, zusätzlich überweist einem der Arbeitgeber die Differenz zwischen Mutterschaftsgeld und dem vorherigen Nettogehalt. Während des Mutterschutzes bekommt man also das gleiche Geld überwiesen wie in der Zeit davor und muss keine finanziellen Einbußen hinnehmen.

Originalquelle

Lukas Hoffmann für Operation Karriere - Das Karriereportal und der Kongress für angehende und junge Ärzte, www.operation-karriere.de $\rightarrow$ Karriereweg $\rightarrow$ Assistenzarzt (Direktlink für Onlineversion: www.operation-karriere.de/karriereweg/assistenzarzt/schwangerschaft-in-der-weiterbildung.htm/). 


\section{Ärztliche Schweigepflicht}

Wann Ärzte Auskunft erteilen dürfen - und wann nicht

Liegt eine gültige Schweigepflichtentbindung des Patienten vor, können Ärzte einem Betreuer oder der Polizei Auskunft erteilen. Ohne diese Entbindung ist die Rechtmäßigkeit sorgfältig im Einzelfall zu prüfen.

Im ärztlichen Alltag fallen viele hochsensible Daten an. Ein diskreter Umgang mit diesen Daten ist daher notwendig, um das Vertrauensverhältnis zwischen Arzt und Patient zu gewährleisten. Dies hat einen derart hohen Stellenwert, dass es durch § 203 des Strafgesetzbuchs und das medizinische Standesrecht geschützt wird. Bereits das Gelöbnis, das zu Beginn der Muster-Berufsordnung für die in Deutschland tätigen Ärztinnen und Ärzte abgedruckt ist, greift die ärztliche Schweigepflicht auf. Jeder Arzt hat die ihm «anvertrauten Geheimnisse auch über den Tod der Patientin oder des Patienten hinaus» zu wahren. Die Verpflichtung zur Verschwiegenheit ist jedoch nicht immer so einfach einzuhalten. Es gibt durchaus rechtlich legitimierte Ausnahmen, die ein Brechen der ärztlichen Schweigepflicht erfordern.

\section{Anfrage des Vormundes oder Betreuers}

Immer wieder erreichen Ärzte Anfragen mit der Bitte um Auskunft oder gar Akteneinsicht. Verlangt der Patient selbst Einsicht in seine Krankenakte, muss ihm dies in der Regel ohne Einschränkung gewährt werden, wenngleich er idealerweise in einem Arzt-PatientenGespräch Einsicht nehmen sollte. Auch wenn ein gesetzlich bestellter Vormund oder Betreuer mit diesem Anliegen an den Arzt herantritt, hat er ein Recht auf Akteneinsicht. Ist beispielsweise ein Volljähriger aufgrund einer psychischen Krankheit oder einer körperlichen, geistigen oder seelischen Behinderung nicht in der Lage, seine Angelegenheiten ganz oder teilweise zu erledigen, kann das Betreuungsgericht einen Betreuer als gesetzlichen Vertreter bestellen. Die Betreuung ist immer auf jene Aufgaben beschränkt, in denen sie erforderlich ist. Mögliche Aufgaben sind Vermögensfürsorge, Postkontrolle, Aufenthaltsbestimmungsrecht oder Gesundheitsfürsorge. Ein Betreuer hat nur Recht auf Auskunft oder Akteneinsicht beim Arzt, wenn die Auskunft für die Wahrnehmung seiner konkreten Aufgabe nötig ist und zwar nur im erforderlichen Umfang. So benötigt ein Betreuer im Aufgabenkreis Vermögensfürsorge beispielsweise keine vollumfängliche Einsicht in die Patientenakte. Das Recht auf Auskunft eines Betreuers kann zusätzlich durch den Willen des Patienten eingeschränkt werden, sofern er einsichtsfähig ist. Eine Offenbarung von Patientengeheimnissen gegen den Willen eines einsichtsfähigen betreuten Patienten ist nicht zulässig. Daher sollte jede Anfrage eines Betreuers vorab sorgfältig geprüft werden.

\section{Anfrage von Ermittlungsbehörden und Gerichten}

Anfragen von Ermittlungsbehörden oder Gerichten erzeugen meist eine besonders hohe Unsicherheit. Man möchte einerseits keine personenbezogenen Daten unbefugt offenbaren, gleichzeitig jedoch die Ermittlungsarbeit der Behörden, wie die Suche nach vermissten Personen oder Unfallopfern, nicht behindern. Grundsätzlich folgt aus der strafrechtlich fixierten ärztlichen Schweige- pflicht (§ 203 StGB) ein Zeugnisverweigerungsrecht für Ärzte sowie deren Berufshelfer (§53 Abs. 1 Nr. 3 und $\S 53$ a StPO) gegenüber den Ermittlungsbehörden und vor Gericht.

Das im November 2015 in Kraft getretene Bundesmeldegesetz berechtigt Krankenhäuser jedoch dazu, gegenüber den zuständigen Behörden Auskunft in bestimmtem Umfang zu erteilen. Dieser umfasst Vor- und Familiennamen, Geburtsdatum und -ort (bei Geburten im Ausland auch den Staat), Staatsangehörigkeit, Anschrift sowie das Datum der Aufnahme und der Entlassung. Die Krankenhäuser dürfen Auskünfte jedoch nur erteilen, wenn dies zur Abwehr einer erheblichen und gegenwärtigen Gefahr, zur Verfolgung von Straftaten oder zur Aufklärung des Schicksals von Vermissten und Unfallopfern erforderlich ist. Den konkreten Grund für das Auskunftsersuchen muss die anfragende Behörde im Einzelfall darlegen. Eine vom Patienten verfügte Auskunftssperre greift in diesem Fall nicht. Jedoch dürfen Ärzte darüber hinaus auch gegenüber der Polizei, Staatsanwaltschaft oder den Gerichten keinerlei Auskünfte über Patienten erteilen.

Anders verhält es sich, wenn der Patient den Arzt von der Verpflichtung zur Verschwiegenheit gegenüber der Polizei oder der Staatsanwaltschaft entbunden hat. Dann müssen Ärzte Auskunft erteilen. Sie ist jedoch auf den in der Entbindungserklärung festgelegten Umfang zu beschränken. Schweigepflichtentbindungen von Angehörigen sind grundsätzlich unwirksam, selbst wenn der Patient bereits verstorben ist. Dennoch können Angehörige und Erben nach $§ 630 \mathrm{~g}$ Abs. 3 BGB ein Recht auf Einsicht in die Patientenakten haben.

\section{Wenn Gefahr im Verzug ist}

Das Zeugnisverweigerungsrecht wird durch das Beschlagnahmeverbot (§ 97 StPO) auf alle Aufzeichnungen, Mitteilungen und Gegenstände der ärztlichen Untersuchungsbefunde erweitert, die sich im Gewahrsam des Krankenhauses befinden. Dazu zählt auch die Patientenakte. Als Folge darf das Krankenhaus Unterlagen nur herausgeben, wenn entweder der Patient eine Entbindung von der Schweigepflicht vorgenommen hat oder die Ermittlungsbehörden einen Beschlagnahmebeschluss vorweisen, den in der Regel nur ein Richter ausstellen kann. Ausnahme: Wenn durch den Zeitverlust beim

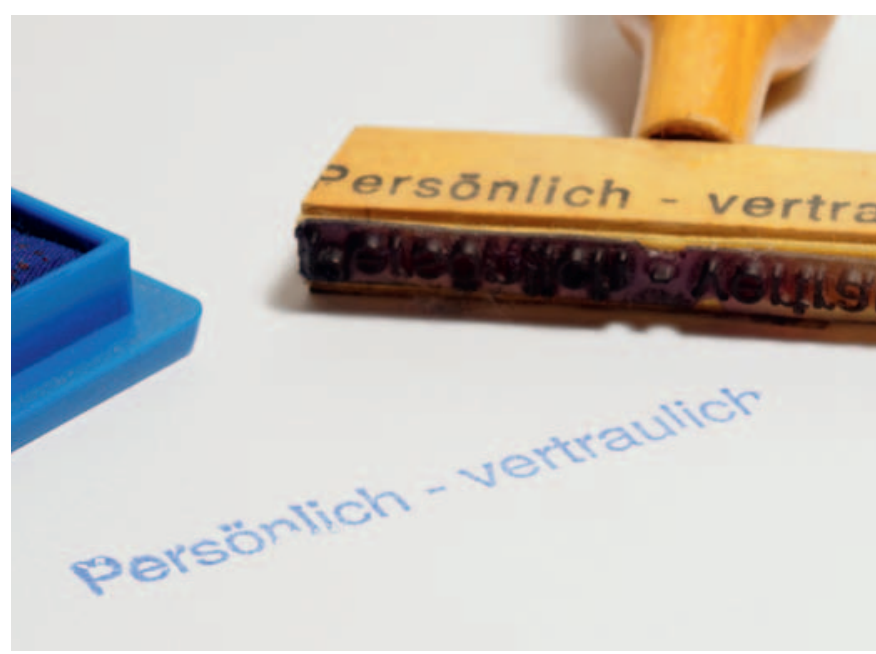


Einholen einer richterlichen Genehmigung Beweismittel verloren gehen können, ist «Gefahr im Verzug». Dann sind auch Polizei oder Staatsanwaltschaft berechtigt, eine Beschlagnahme anzuordnen. Liegt ein gültiger Beschlagnahmebeschluss vor, müssen die Unterlagen im Original herausgegeben werden. Dabei gilt, unbedingt den genauen Umfang der Anordnung zu beachten, also auf welche Unterlagen sich diese konkret bezieht. Um die Patientensicherheit zu gewährleisten, sollten von allen Dokumenten Kopien angefertigt werden, bevor die Unterlagen herausgegeben werden. Zusätzlich sollten im Beschlagnahmeverzeichnis alle herausgegebenen Dokumente vollständig aufgeführt sein.

\section{Widerspruch gegen die Beschlagnahme}

Wenn die Ermittlungsbehörden keinen Beschlagnahmebeschluss vorlegen können, sollten Krankenhausmitarbeiter der Herausgabe ausdrücklich widersprechen und auf eine Dokumentation des Widerspruchs im Beschlagnahmeprotokoll bestehen. Fordern die Behörden dennoch die Herausgabe der Unterlagen, muss das Krankenhaus die Beschlagnahme dulden. Die Mitarbeiter sollten den Beamten dann lediglich zeigen, wo die Unterlagen lagern, diese jedoch nicht aktiv herausgeben.

\section{Zu den Autoren}

Dr. rer. nat. Henning Kropp ist Senior Consultant der Sanovis $\mathrm{GmbH}$. Vorher promovierte er am Institut für anorganische und allgemeine Chemie an der Friedrich-Alexander-Universität Erlangen-Nürnberg.

Dr. sc. hum. Uwe Günther ist geschäftsführender Gesellschafter der Sanovis GmbH. Als Diplom-Informatiker, Diplom-Wirtschaftsingenieur und Gesundheitswissenschaftler blickt er auf über 20 Jahre Erfahrung im Bereich Consulting zurück

\section{Originalquelle}

Kropp H, Günther U: Ärztliche Schweigepflicht: Wann Ärzte Auskunft erteilen dürfen - und wann nicht. Dtsch Arztebl 2017:114;48. (Direktlink zur Originalversion: www.aerzteblatt.de/archiv/195023/Aerztliche-Schweigepflicht-Wann-Aerzte-Auskunfterteilen-duerfen-und-wann-nicht

Kontaktadresse: Dr. sc. hum. Uwe Günther, Sanovis GmbH, Richard-StraussStraße 69, 81679 München, Deutschland, info@ sanovis.com. 
Wissenschaftliches Publizieren

(Warum) brauchen wir Verlage?

Die Aufgaben eines Verlags werden immer sein: Das Sammeln, Sichten, Auswählen, Verdichten, Verbreiten und Bewahren von Informationen in allen Formen und auf jede erdenkliche Weise. Dies sagte schon mein Urgroßvater vor 127 Jahren bei der Gründung des Karger Verlags. Und das gilt noch heute: Wissenschaftliche Verlage haben mehr denn je die Aufgabe des Vermittelns und Vernetzens zwischen den verschiedenen Akteuren der Wissenschaft. Sie selektieren Inhalte und bieten damit Orientierung in einer immer größer werdenden Flut an Informationen. Die Verlage organisieren das Peer Reviewing, das eine wissenschaftlich hochstehende Qualität gewährleistet. Außerdem brinwahren der Informationen, das Archivieren, gerade in der digitalisierten Wissensgesellschaft. Ein weitgehend «unsichtbarer» Service gen Verlage den Inhalt durch Lektorat und Layout in eine stringente und ansprechende Form. Ein wichtiger Aspekt ist auch das Be-

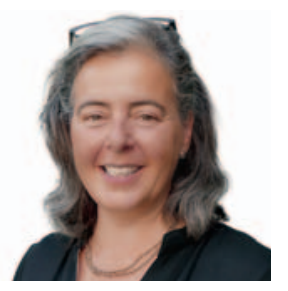

Gabriella Karger Delegierte des Verwaltungsrats \& Verlegerin, Karger $A G$ schaftlichen Diskurs und Erkenntnisfortschritt und sind dadurch sowohl Dienstleister als auch Partner der Wissenschaft.

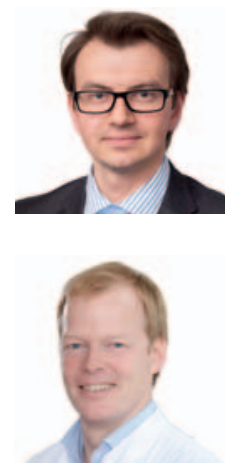

_ Prof. Dr. Matus Rehak

Herausgeber Karger Kompass Ophthalmologie

Ja, denn so kann jeder Wissenschaftler seine Untersuchungen einreichen und sicher sein, dass diese durch ein unabhängiges Begutachtungsverfahren (Peer-review) geprüft werden. Außerdem muss ich mich als Autor nicht mit den verlegerischen Aspekten, wie Lektorat, Gestaltung etc. beschäftigten, sondern ich weiß, dass der Verlag sich kümmert.

\section{—Prof. Dr. Nicolas Feltgen}

\section{Fachbeirat Karger Kompass Ophthalmologie}

Ja, wir brauchen Verlage, denn sie sind in der Lage, Informationen entsprechend den Interessen der Leser und Autoren zu bündeln. Die Qualität dieser Beiträge wird zudem durch ein Peer-Review geleistet, das von den Verlagen organisiert und koordiniert wird. Dadurch wird nicht zuletzt die wissenschaftliche Qualität auf einen Nenner und vergleichbar gemacht. Insbesondere bei KARGER KOMPASS wird die Qualität der Arbeit durch die kritische Auseinandersetzung im Wissenstransfer auf einer praxisorientierten Ebene geprüft. So werden auch über Verlage Diskussionen angeregt, die über den rein wissenschaftlichen Kontext hinausgreifen.

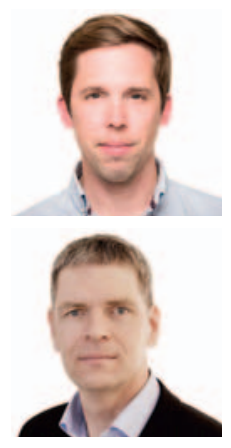

— Dr. Matthias Raspe

Koordination «PneumoCampus», Karger Kompass Pneumologie

Bei der unüberschaubaren Menge und Fluktuation von Fachinformationen, wie wir sie heute vorfinden, sind Verlage von unschätzbaren Wert. Sie ordnen und bereiten Fachwissen auf und machen es somit erst wirklich nutzbar.

\section{Bernd Adam}

Geschäftsführer Deutsche Fachpresse

Die heutige Wissenschafts- und Arbeitswelt ist dadurch gekennzeichnet, dass Informationen im Überfluss vorhanden sind und die Menge rasant wächst. Welche Informationen aber sind relevant für den einzelnen Menschen? Wie sind die Informationen zu bewerten? Und worauf ist wirklich Verlass? Verlage bieten mit ihren Produkten fundierte Einordnung durch Expertise, wissenschaftliche Standards und hohe journalistische Qualität. Sie machen aus Informationen relevantes Wissen und geben Orientierung. Dabei sind sie nicht nur als verlässliche Informationsquellen, sondern auch als unabhängige Meinungsträger essenziell. Kurz: Ja, wir brauchen Verlage, denn ihre Arbeit ist unverzichtbarer Bestandteil einer wissensstarken und freien Gesellschaft.

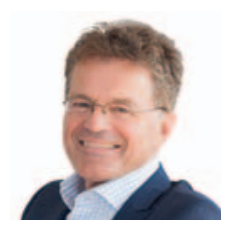

\section{_Alexander Skipis}

Hauptgeschäftsführer des Börsenvereins des Deutschen Buchhandels

Verlage sind Garanten für die Qualität und Vielfalt des deutschen Buchmarktes und tragen wesentlich zum Gelingen einer freien, demokratischen Gesellschaft bei. Verlage unterstützen Autorinnen und Autoren im gesamten Veröffentlichungsprozess, sie geben ihren Ideen eine Form und bringen sie gestützt durch Lektorat, Herstellung, Marketing und Vertrieb an den Leser. Mit ihrer Arbeit tragen Verlage zur Verbreitung von Ideen und zur Meinungsvielfalt bei. Sie stoßen Debatten an und leisten damit einen wichtigen Beitrag zum kulturellen und gesellschaftlichen Leben. 